\title{
Regulation of expression and catalytic activity of Escherichia coli RsmG methyltransferase
}

\author{
ALFONSO BENÍTEZ-PÁEZ, ${ }^{1,2}$ MAGDA VILLARROYA, ${ }^{1}$ and M.-EUGENIA ARMENGOD ${ }^{1,3}$ \\ ${ }^{1}$ Laboratorio de Genética Molecular, Centro de Investigación Príncipe Felipe, 46012 Valencia, Spain \\ ${ }^{2}$ Bioinformatic Analysis Group-GABi, Centro de Investigación y Desarrollo en Biotecnología, Bogotá D.C. 111221, Colombia
}

\begin{abstract}
RsmG is an AdoMet-dependent methyltransferase responsible for the synthesis of $\mathrm{m}^{7} \mathrm{G527}$ in the 530 loop of bacterial $16 \mathrm{~S}$ rRNA. This loop is universally conserved, plays a key role in ribosomal accuracy, and is a target for streptomycin binding. Loss of the $\mathrm{m}^{7} \mathrm{G} 527$ modification confers low-level streptomycin resistance and may affect ribosomal functioning. Here, we explore the mechanisms controlling RsmG expression and activity, which may somehow respond to the demand set by the amount of rRNA. We confirm that rsmG is the second member in a bicistronic operon and demonstrate that rsmG also has its own promoter, which appears, in actively growing cells, as a control device to offset both the relatively low stability of RsmG and inhibition of the operon promoter. RsmG levels decrease under conditions that down-regulate rRNA synthesis. However, coordination between rRNA and RsmG expression does not seem to occur at the level of transcription initiation. Instead, it might depend on the activity of an inverted repeated region, located between the rsmG promoter and ribosome binding site, which we show to work as a weak transcriptional terminator. To gain insights into the enzymatic mechanism of RsmG, highly conserved residues were mutated and the abilities of the resulting proteins to confer streptomycin resistance, to modify rRNA, and to bind AdoMet were explored. Our data demonstrate for the first time the critical importance of some residues located in the active site of Escherichia coli RsmG for the $\mathrm{m}^{7} \mathrm{G}$ modification process and suggest a role for them in rRNA binding and catalysis.
\end{abstract}

Keywords: rRNA modification; transcriptional regulatory elements; RsmG stability; mutational analysis; catalytic mechanism

\section{INTRODUCTION}

RsmG is an AdoMet-dependent methyltransferase responsible for the synthesis of $\mathrm{m}^{7} \mathrm{G} 527$ in the 530 loop of bacterial 16S rRNA (Okamoto et al. 2007). The 530 loop region is universally conserved and appears to play a key role in ribosomal accuracy (Ogle et al. 2003; Ogle and Ramakrishnan 2005). During tRNA selection, rotation of the 530 loop modulates interactions between ribosomal regions that, when mutated, either increase or decrease mRNA misreading. Moreover, streptomycin binds to $16 \mathrm{~S}$ rRNA in four different domains, one including nucleotides G526 and G527 in the 530 loop, thus perturbing the A-site function and leading to translational misreading (Carter et al. 2000; Ogle and Ramakrishnan 2005). In spite of participation of the 530 loop in the decoding process being clear, the precise role of the $\mathrm{m}^{7} \mathrm{G} 527$ modification has remained elusive. Loss of RsmG activity confers low-level

\footnotetext{
${ }^{3}$ Corresponding author.

E-mail armengod@cipf.es.

Article published online ahead of print. Article and publication date are at http://www.rnajournal.org/cgi/doi/10.1261/rna.029868.111.
}

streptomycin resistance and inexplicably prompts the appearance of high-level streptomycin resistance mutations in the S12-encoding gene, rpsL (Nishimura et al. 2007a,b; Okamoto et al. 2007; Gregory et al. 2009; Wong et al. 2011). Moreover, the effects of rsmG mutations on bacterial fitness and translational accuracy have been reported as being variable, depending on the bacterial species (Nishimura et al. 2007b; Okamoto et al. 2007; Gregory et al. 2009; Ochi et al. 2009). We recently found that a null $r s m G$ mutation produces an error-prone phenotype in certain genetic backgrounds, which suggests that modification $\mathrm{m}^{7} \mathrm{G} 527$ is important for ribosomal accuracy and that pleitropy may explain, at least partially, the observed variability (A Benítez-Páez, M Villarroya, and M-E Armengod, in prep.).

To ensure proper modification of rRNA, it seems reasonable that regulation of $r s m G$, the RsmG encoding gene, correlates somehow with rRNA synthesis, which is linked to several global regulatory networks and so carefully balanced in response to changing growth conditions (Keener and Nomura 1996; Magnusson et al. 2005; Potrykus and Cashel 2008; Jin et al. 2011). Particularly, rRNA synthesis is strongly down-regulated by the alarmone ppGpp, whose levels increase under nutrient-limitation conditions and circumstances that 
cause growth arrest. Due to previous intensive studies, we have a fairly complete picture of the mechanisms controlling rRNA synthesis. However, information on the mechanisms regulating the expression of rRNA modification enzymes, including RsmG, is scarce.

The Escherichia coli rsmG gene is thought to be the second member in a bicistronic operon headed by $m n m G$, which is adjacent to the chromosome replication origin, oriC (von Meyenburg et al. 1982). The protein encoded by $m n m G$ is involved, together with the MnmE GTPase, in tRNA modification (Moukadiri et al. 2009). Transcription of $m n m G$ starts at a site $63 \mathrm{bp}$ from the left end of oriC and proceeds leftward away from it, reading into the $m n m G$ and rsm $G$ genes (formerly designated as gidA and gidB, respectively). Since $r s m G$ expression was seen to be impaired by $\operatorname{Tn} 10$ insertions in the $m n m G$ gene, $r s m G$ was proposed to be cotranscribed with $m n m G$ (Hansen et al. 1981). Transcription from the $m n m G$ promoter $(m n m G p)$ has been shown to be involved in the initiation of chromosome replication (Ogawa and Okazaki 1991). It is most active before initiation of replication but is dramatically inhibited after this step during the time corresponding to the sequestration period of the oriC region from the dam methyltransferase (Theisen et al. 1993; Ogawa and Okazaki 1994). Moreover, $m n m G p$ activity is inhibited by ppGpp in vitro (Ogawa and Okazaki 1991). Regulation of the distal gene of the operon, $r s m G$, has not been specifically investigated and still requires further study. Genomic expression analyses have reported a fivefold higher transcriptional expression of rsmG if compared to $m n m G$ (Bernstein et al. 2002; data available at http://chase.ou.edu/oubcf/), whereas a largescale study on the abundance of proteins in E. coli has determined that the number of molecules per cell of RsmG and MnmG is similar (Ishihama et al. 2008). Therefore, additional regulatory mechanisms, other than the transcription from $m n m G p$, are likely to control the expression of rsm $G$ (and $m n m G$ ).

RsmG-mediated modification of RNA may depend not only on the RsmG concentration within the cell but also on the catalytic activity of the enzyme. RsmG belongs to Class I methyltransferases (Schubert et al. 2003). Its structure shows a common Rossmann fold where the characteristic AdoMet binding signature motif $\mathrm{GxGxG}$ is found at the end of the first $\beta$-strand (Romanowski et al. 2002; Gregory et al. 2009). However, the structural basis for substrate recognition and catalysis by RsmG (as for most $m^{7} G$ MTases) remains poorly characterized (Purta et al. 2005; Gregory et al. 2009; Husain et al. 2010). Structures of E. coli and Bacillus subtilis RsmG have been determined in the apo form (Romanowski et al. 2002; and PDB id 1XDZ, respectively), whereas RsmG protein from Thermus thermophilus has been crystallized both in the apo form and complexed with AdoMet and AdoHcy (Gregory et al. 2009). One of the complexes of $T$. thermophilus RsmG with AdoMet also contained adenosine monophosphate (AMP), suggesting a putative RNA-binding site in the C-terminal region, remote from the active site. The $T$. thermophilus and E. coli RsmG proteins differ in substrate preference: the first recognizes naked $16 \mathrm{~S}$ rRNA, whereas E. coli RsmG has been reported to require the intact $30 \mathrm{~S}$ subunit for methylation in vitro (Okamoto et al. 2007; Gregory et al. 2009). The E. coli RsmG apo-enzyme structure lacks the C-terminal region present in T. thermophilus RsmG, which provides the AMP-binding site. This could partly explain the differences observed in substrate preference terms and indicate a different mode of substrate interaction between the two enzymes (Gregory et al. 2009). A comparison of the active site region has revealed that key residues are conserved between T. thermophilus and E. coli RsmG, although some differences were noted (Gregory et al. 2009).

The aim of this study is to explore the regulatory mechanisms that modulate the $\operatorname{rsm} G$ expression at transcriptional and post-transcriptional levels, as well as to identify amino acid residues responsible for catalysis of the methylation reaction. We show that $r s m G$ has its own promoter ( $r s m G p$ ) and that protein RsmG is almost twofold less stable than MnmG. These results suggest that the rsmGp activity may compensate both the inhibition of the operon promoter $(m n m G p)$ after replication initiation at oriC and the relatively low stability of RsmG. Interestingly, our results support that the coordinate expression of RsmG and rRNA does not occur at the transcription initiation level. Rather, other regulatory mechanisms acting in later steps appear to be involved in adjusting the RsmG expression to rRNA amounts. Finally, we demonstrate the critical importance of some residues in or around the active site of E. coli RsmG for the $\mathrm{m}^{7} \mathrm{G}$ modification process and propose a role for them in rRNA binding and catalysis.

\section{RESULTS AND DISCUSSION}

\section{Prediction of novel DNA elements controlling the $E$. coli rsmG expression}

By using integrative genomic tools (von Mering et al. 2007) to explore both the genomic context and the co-occurrence profiles of $r s m G$, we found that in most of the bacterial genomes where $r s m G$ is present, it is clustered with $m n m G$. Gene $m n m G$ was originally called gidA because of the glucose inhibited division phenotype (Gid) observed after its inactivation by Tn10 insertions (von Meyenburg et al. 1982). On rich medium plates containing glucose, the mutant cells were considerably elongated, indicating division inhibition through a mechanism that remains to be investigated. It probably involves the translational control of some division-related protein, given that MnmG has been shown to be a tRNA modification enzyme (Moukadiri et al. 2009), and no evidence for a direct role of MnmG in cell division has been reported so far. According to the nomenclature originally proposed for the first operon gene, 
$r s m G$ was designated as gidB even though its inactivation by Tn10 insertions did not produce the Gid phenotype (von Meyenburg et al. 1982). To further explore the regulation of both RNA modification enzymes, we first analyzed the native expression of RsmG and MnmG in different strains carrying mini-Tn10 (Bregeon et al. 2001) or $\mathrm{kan}^{R}$ (Baba et al. 2006) insertions in $m n m G$ (Fig. 1). As expected, no protein corresponding to $\mathrm{MnmG}$ was detected in the different truncated mnmG mutants (Fig. 1B). In contrast, accumulation of RsmG was always observed but at different levels, depending on the inserted element in the preceding gene (see mutants IC5933, IC5934, IC5935, and IC5936 in Fig. 1B). The lower amounts of RsmG detected in IC5934 and IC5935 (where Tn10 insertion occurs at the beginning of $m n m G$ ) are in agreement with the proposal that $r s m G$ forms an operon with $m n m G$ and, therefore, that its transcription greatly depends on the $m n m G$ promoter (mnmGp) (von Meyenburg et al. 1982). Unlike the results found with the mnmG::Tn10 mutants, RsmG accumulated in the $m n m G:: k a n$ mutant (IC5936) at similar levels to the wild-type strain, suggesting that $r s m G$ transcription is mediated in IC5936 by a promoter located within the $\mathrm{Kan}^{\mathrm{R}}$ cassette and/or by mnmGp (still present in this mutant). The lower RsmG expression in the $m m m G:: \operatorname{Tn} 10$ mutants could thus be due to stronger polar effects of miniTn 10 on transcription mediated by $m n m G p$, the activity of a promoter within mini-Tn10, or a promoter located in the C-terminal region of $m n m G$. We decided to explore the latter hypothesis given that the discovery of new transcrip-

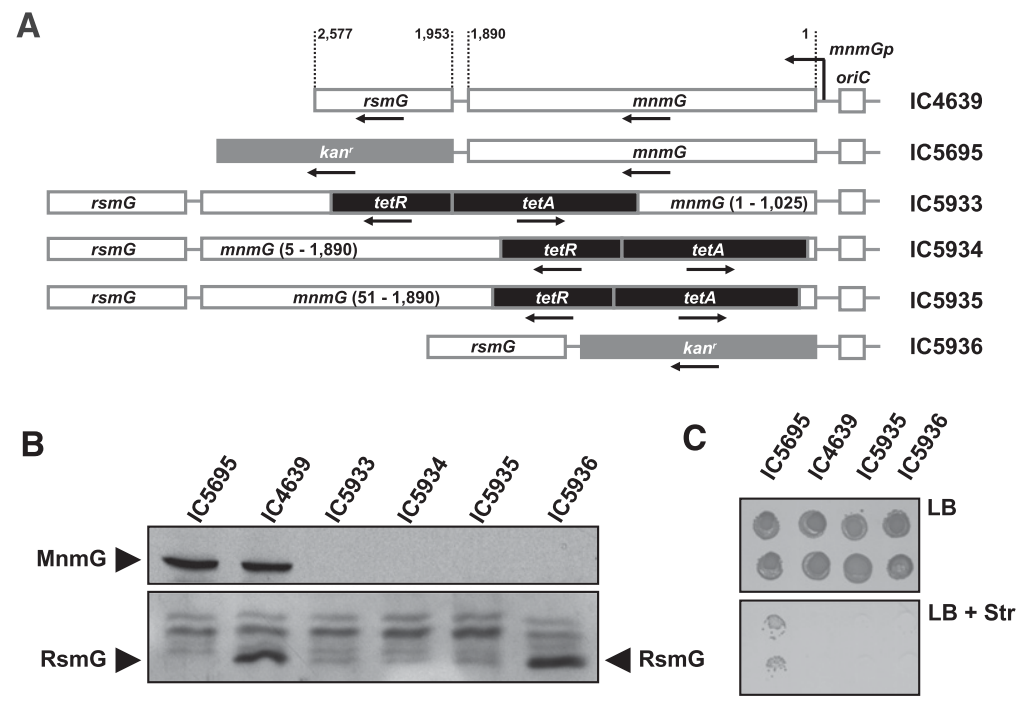

FIGURE 1. Expression of RsmG in $m n m G$ chromosomal mutants. (A) Genetic organization of the different $m n m G$ and $r s m G$ chromosomal mutants used in this study. Black arrows denote gene and transcription orientation. Mini Tn10 and $\mathrm{kan}^{r}$ elements are colored in black and gray, respectively. The limits of the $m n m G$ and $r s m G$ structural genes and the positions of $m n m G p$ and oriC are indicated on the IC4639 chromosomal map (top). In the IC5933, IC5934, and IC5935 maps, numbers inside the mnmG ORF inform about the insertion point of the mini-Tn10 element. (B) Immunoblotting, using anti-MnmG and anti-RsmG, of extracts from the indicated strains. $(C)$ Growth of the wild-type strain (IC4639) and some selected insertion mutants on LAT plates supplemented with $20 \mu \mathrm{g} / \mathrm{mL}$ streptomycin. tional elements of the $m n m G-r s m G$ operon is relevant for understanding regulation of MnmG and RsmG activity. In this respect, it should be noted that, in spite of the low RsmG levels accumulating in the mnmG::Tn10 strains, these mutants exhibit a wild-type phenotype; i.e., they are streptomycin-sensitive (Fig. 1C).

An in silico search for putative promoters in a 3-kb DNA sequence containing the mnmG-rsmG operon was performed by two different algorithms (see Materials and Methods). In both cases, two regions with a high probability of including a promoter sequence were detected (Fig. 2A). The first ( -92 to -115 , in relation to the $m n m G$ start codon) contains the previously identified $m n m G$ promoter ( $m n m G p$ ) (Kolling et al. 1988); the second lies in the $3^{\prime}$ region of $m n m G$, around position 1881 or 1885 , thus supporting the hypothesis that $r s m G$ expression in the $m n m G:: \operatorname{Tn} 10$ mutants is at least partially mediated by its own promoter $(r s m G p)$. Interestingly, $r s m G p$ is located upstream of an inverted repeated region mapped between $m n m G$ and rsm $G$ (Walker et al. 1984). This inverted repetition is able to generate a very stable stem-loop secondary structure (Fig. 2A), which might work as a transcriptional pause signal.

\section{Activity of the predicted transcription signals}

To assess the functional role of the predicted regulatory signals in $r s m G$ expression, we made use of transcriptional fusions to the lacZ reporter gene (Macian et al. 1994). As shown in Figure $2 \mathrm{~B}$, the fragments carried by pIC1371 and pIC1373, which contain the putative rsmGp region, promote lac $Z$ transcription, although activity is about threefold lower than that associated with the $m n m G p$ region (pIC1374). Moreover, rsmGp activity diminishes by about fourfold when the region between positions 1891 and 1953, which contains the inverted repeat, is also included in the insert (pIC1372). This supports the idea that the predicted stem-loop ( $t$, hereafter) may work as a weak transcriptional terminator. By splitting the DNA region carried by pIC1373 and cloning the resulting fragments, we constructed pIC1460 (positions 1838 to 1890 ) and pIC1461 (positions 1739 to 1842), and determined that the transcriptional activity is associated with a short 53-bp region that extends between positions 1838 and 1890 (Fig. 2B). Within this region, the most probable sequences corresponding to boxes -10 and -35 are depicted in Figure 2A. Note that, whereas 


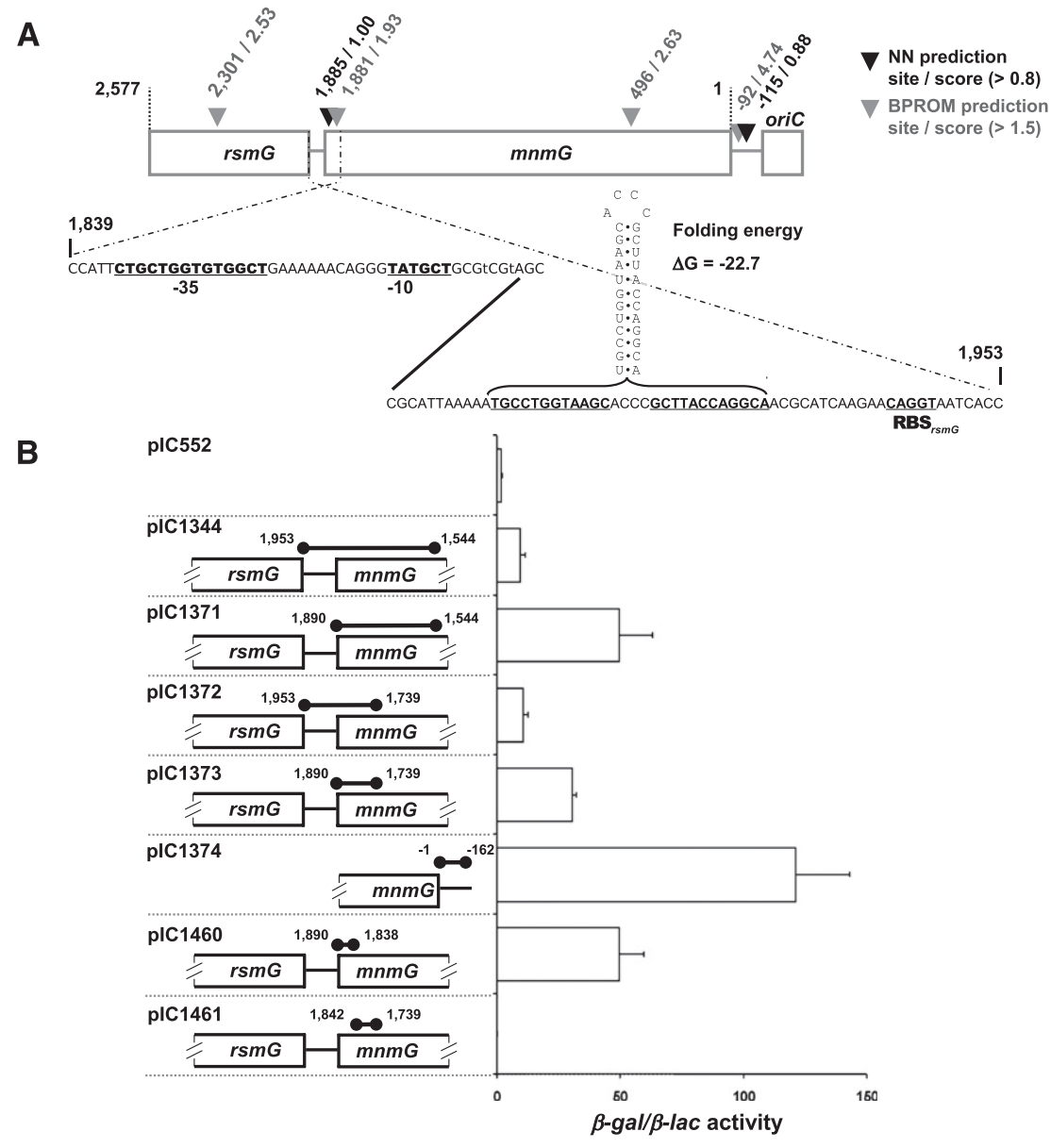

FIGURE 2. Characterization of the $r s m G$ regulatory elements. (A) Prediction of new DNA elements regulating $r s m G$ expression. The top of the figure shows a schematic of the chromosomal region including the $m n m G-r s m G$ operon and the predictions of the promoter regions by the Neural Network Promoter Prediction (black) (Reese 2001) and BPROM (gray) (http://www.softberry.com/berry.phtml) servers. Below the schematic, the sequence of the region comprising the $3^{\prime}$ end of $m n m G$ and the $m n m G-r s m G$ intergenic region is shown. Numbers flanking the sequence indicate sequence positions. Nucleotides in bold and underlined show the Ribosome Binding Site (RBS) of $r s m G$ and the complementary sequences that can form a potentially stable stem-loop structure in the $5^{\prime}$ region of the $r s m G$ mRNA (Walker et al. 1984). Free energy calculated according to Zuker (2003). The most probable -10 and -35 boxes of the $r s m G$ promoter are also indicated. (B) Schematic representations of the DNA fragments cloned into pIC552 (left) and the transcriptional activity $(\beta-\mathrm{Gal} / \mathrm{Bla})$ of the resulting fusions (right) in MC1000. Black bars and numbering at top of each diagram of the $m n m G-$ rsm $G$ operon denote the region inserted into pIC552.

the -10 box agrees with the consensus Pribnow box (TATAAT) in four of the six nucleotides, the -35 region still offers a poorer fit to the consensus sequence (TTGACA).

Finally, rsmGp activity was also investigated by a complementation assay using an $r s m G$ null mutant. We inserted a DNA fragment containing the structural $r s m G$ gene with or without $r s m G p$ into pIC552, downstream from the $\Omega$ fragment, which severely blocks transcription from vector promoters (Macian et al. 1994). Full complementation $(97 \%)$ was observed with a construct carrying rsmGp-tt$r s m G$, but not with a plasmid containing tt-rsmG, where partial complementation (11\%) was probably due to residual transcription from plasmid promoters (Supplemental Fig. S1).

Genomic expression analyses have reported a fivefold higher transcriptional expression of $r s m G$ compared to $m n m G$ (Bernstein et al. 2002). This difference might be due to selective degradation of the $m n m G$ region in the bicistronic mRNA or to the presence of a specific promoter for $r s m G$. The data presented herein lend support to the latter hypothesis.

Curiously, activity of $r s m G p$ slightly increased (about fourfold) when cells were grown in minimal media supplemented with glycerol, a poor carbon source, instead of glucose (Table 1), or when cells growing in LBT entered into the stationary phase (Table 2); i.e., induction of rsmGp occurs under stress conditions known to stimulate ppGpp accumulation and down-regulate rRNA synthesis. The slight induction of $r s m G p$ during stationary phase was independent of the stress-inducible sigma factor RpoS (also named $\sigma^{38}$ ), since it was also observed in an rpoS mutant (Table 2). It is then possible that $r s m G p$ is subjected to passive regulation, being activated because of an increased availability of $\sigma^{70}$ bound RNA polymerase under circumstances that restrict growth (Gummesson et al. 2009). In any case, our results support that expressions of RsmG and rRNA are not coordinated at the level of transcription initiation. However, other mechanisms may be responsible for adjusting RsmG expression to rRNA synthesis. For example, ppGpp is known to enhance pausing of RNA polymerase during transcription elongation and to inhibit translation initiation by interacting with the initiation factor IF2 (Magnusson et al. 2005; Srivatsan and Wang 2008; Jin et al. 2011). The location of the transcriptional pause signal $(t t)$ between $r s m G p$ and the ribosome binding site (RBS) of rsmG (see Fig. 2A) makes this regulatory element a good candidate for coordinating expression of the rRNA and $r s m G$ genes. We have found that the levels of RsmG are reduced in the stationary phase of growth with respect to the exponential phase, or in cells growing in minimal medium containing a poor carbon source (glycerol) compared to cells growing in the presence of glucose (Fig. 3A). The fact that the RsmG levels decrease under conditions where we have observed an increased $r s m G p$ activity 
TABLE 1. Effect of the carbon source on the $r s m G p$ and $m n m G p$ transcriptional activity

\begin{tabular}{lllrr}
\hline & & & \multicolumn{2}{c}{ Bgal/Bla units $^{\text {a }}$} \\
\cline { 4 - 5 } Strain & Plasmid & Promoter & M9glu & M9gly \\
\hline MC1000 & plC1373 & rsmGp & 33.6 & 100.7 \\
MC1000 & pIC1374 & mnmGp & 264.2 & 455.0 \\
BW25113 & plC1373 & rsmGp & 21.9 & 105.9 \\
BW25113 & plC1374 & mnmGp & 315.5 & 618.0 \\
\hline
\end{tabular}

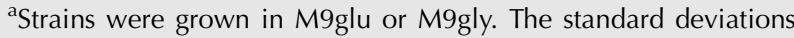
were $\sim 10 \%$ of the average values.

(Tables 1 and 2) supports that some mechanism(s) acting after transcription initiation modulate(s) the RsmG expression. Further experiments are required to test this hypothesis and to clarify the role of the transcriptional pause signal.

\section{RsmG has a shorter half-life than MnmG}

A large-scale study on protein abundance in E. coli has determined that the number of molecules per cell of RsmG and MnmG is similar (Ishihama et al. 2008). This apparently contrasts with the results showing that $r s m G$ transcripts are about fivefold more abundant than $m n m G$ transcripts (Bernstein et al. 2002). Both studies were performed with cultures in the exponential phase of growth ( $\left.\mathrm{OD}_{600} \sim 0.5-0.8\right)$. Therefore, it is reasonable to assume that in actively growing cells, translational or post-translational mechanisms control accumulation of the RsmG protein. We found that RsmG is almost twofold less stable than MnmG (Fig. 3B), which helps to fit the data from transcriptional and proteomic analyses. Taken together, our results suggest that the $r s m G$ promoter may work as a control device to offset both the relatively lower stability of RsmG and the inhibition of the operon promoter $(m n m G p)$ after replication initiation at oriC, thus playing an important role during exponential growth.

\section{Identification and characterization of functional residues in $E$. coli RsmG}

To identify residues critical for E. coli RsmG functional activity, we built a sequence profile of the RsmG family from 27 representative sequences of major bacterial groups (see Materials and Methods). Based on both this profile (schematically summarized in Fig. 4A) and structural comparisons (Fig. 4B,C), we predicted specific roles for some conserved residues of E. coli RsmG (Table 3). These residues were mutated to alanines, and the abilities of the resulting proteins to confer streptomycin resistance, to modify $\mathrm{rRNA}$, and to bind AdoMet were explored.
For the in vivo complementation assays (streptomycinresistance and rRNA modification assays), we used strain IC5695 $(\Delta r s m G)$ carrying pBAD-TOPO or pBAD-TOPO derivatives encoding C-terminal FLAG-tagged RsmG proteins. Cells were grown in the absence of the arabinose inducer to achieve recombinant protein expression levels close to the native protein level (Fig. 4D). Samples from cultures grown under the same conditions were used to analyze the production and solubility of RsmG-FLAG proteins (Fig. 4D), streptomycin resistance (Supplemental Fig. S2), and the rRNA modification status (Table 3). As expected, strain IC5695 $(\Delta r s m G)$ harboring an empty vector was streptomycin-resistant on LAT plates containing $20 \mu \mathrm{g} / \mathrm{mL}$ of the antibiotic, whereas expression of the wildtype RsmG-FLAG protein from pIC1343 conferred a wildtype, streptomycin-sensitive phenotype (Table 3; Supplemental Fig. S2). Likewise, $\Delta r s m G$ cells expressing proteins G73A, R123A, and K165A were streptomycin-sensitive, suggesting that these mutations do not greatly impair the RsmG function. In contrast, expression of D56A, D71A, G75A, G77A, P79A, D96A, K100A/R101A, R139A, and R197A conferred a level of resistance similar to that of an rsm $G$ null mutant; cells containing proteins $\mathrm{H} 53 \mathrm{~A}$ and $\mathrm{R} 139 \mathrm{~K}$ were also resistant but to a lesser degree (partial growth on LAT plates containing $20 \mu \mathrm{g} / \mathrm{mL}$ of streptomycin). Therefore, we conclude that all these mutations affect the RsmG function to some extent.

To evaluate the $\mathrm{m}^{7} \mathrm{G}$ methyltransferase activity of the RsmG-FLAG mutant proteins in vivo, $16 \mathrm{~S}$ rRNA was purified from strains expressing the recombinant proteins and treated with nuclease $\mathrm{P} 1$ and alkaline phosphatase. The resulting nucleosides were analyzed by HPLC, and the $\mathrm{m}^{7} \mathrm{G}$ levels were compared to that observed in the strain expressing the wild-type RsmG-FLAG protein. The results shown in Table 3 suggest that the in vivo $\mathrm{m}^{7} \mathrm{G}$ methyltransferase activity is impaired in all mutants, be it to different degrees. Western blot analysis revealed no significant differences in both expression and solubility of the recombinant proteins (Fig. 4D). Therefore, the effects observed

TABLE 2. Effect of the growth phase on the rsmGp and mnmGp transcriptional activity

\begin{tabular}{lllll}
\hline & & & \multicolumn{2}{c}{ Bgal/Bla units $^{\mathrm{b}}$} \\
\cline { 4 - 5 } Strain $^{\mathrm{a}}$ & Plasmid & Promoter & OD $^{\mathrm{c}}<0.3$ & OD $^{\mathrm{c}}>1.7$ \\
\hline MC4100 & plC1373 & rsmGp & 22.5 & 55.3 \\
RH90 & plC1373 & rsmGp & 20.9 & 43.4 \\
MC4100 & plC1374 & mnmGp & 218.7 & 514.8 \\
RH90 & plC1374 & mnmGp & 250.1 & 492.5 \\
\hline
\end{tabular}

${ }^{\mathrm{a}} \mathrm{RH} 90$ is an rpoS mutant derivative of MC4100.

${ }^{\mathrm{b}}$ Strains were grown in LBT.

${ }^{\mathrm{C}}$ Optical density at $600 \mathrm{~nm}$. Each value is the mean of at least two separate experiments. The standard deviations were $\sim 10 \%$ of the average values. 
A

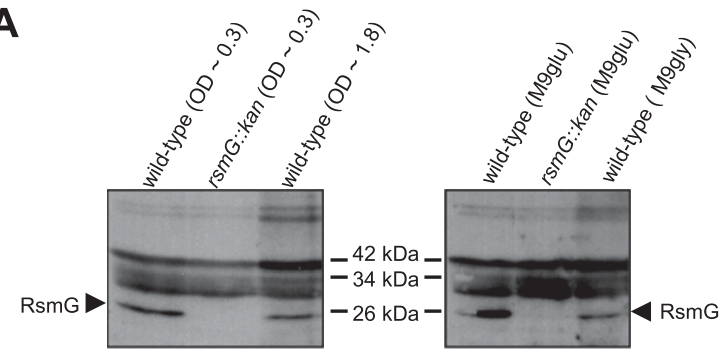

B

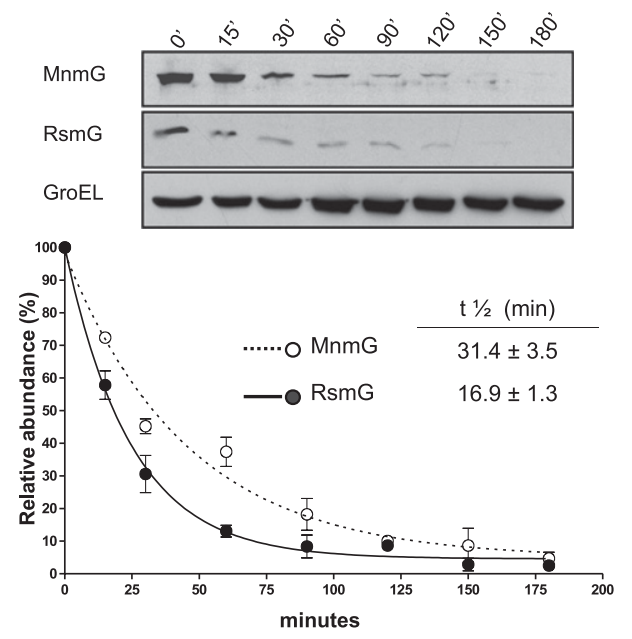

FIGURE 3. Immunoblot analysis of RsmG protein levels. (A) RsmG levels under different growth conditions. Left, cells were grown in LBT until they reached the optical density (OD) at $600 \mathrm{~nm}$ of $\sim 0.3$ or 1.8 . Right, cells were grown in M9glu or M9gly until $\mathrm{OD}_{600} \sim 0.5$. In all cases, cultures were maintained for at least 5-10 generations in exponential growth by repeated dilution with the same medium before being assayed or allowed to continue until stationary phase. Equal amounts of bulk protein $(150 \mu \mathrm{g})$ were loaded in each lane. Note the relative decrease of RsmG in cultures of the wild-type strain at $\mathrm{OD}_{600} \sim 1.8$, in comparison to $\mathrm{OD}_{600} \sim 0.3$ (left), or grown in M9gly, in comparison to M9glu (right). Strains were IC4639 (wild type) and IC5695 (rsmG::kan). (B) Half-life of proteins MnmG and RsmG. At the top, a representative Western blotting assay showing in vivo disappearing of $\mathrm{MnmG}$ and RsmG after glucose addition to cultures of IC5936/pIC1345 and IC5695/pIC1686, respectively. GroEL was used as a loading control. At the bottom, decay curves of MnmG and RsmG derived from densitometric analysis of Western blots. The half-life time for MnmG and RsmG is shown in min \pm SEM from two independent experiments.

on $\mathrm{m}^{7} \mathrm{G}$ synthesis do not appear to result from a lower accumulation of mutant proteins in the cell. In fact, there is a good correlation between the levels of rRNA modification and streptomycin resistance: levels of modification close to $80 \%$ lead to a wild-type phenotype in relation to streptomycin susceptibility, whereas levels of modification below $60 \%$ result in a streptomycin-resistant phenotype.

C-terminal 6His-tagged RsmG proteins, overexpressed from pET15b derivatives, were purified and tested for AdoMet binding by Surface Plasmon Resonance (SPR). As shown in Table 3, the $K_{\mathrm{d}}$ was found to be $0.39 \pm 0.02 \mu \mathrm{M}$ for the wild-type enzyme. Mutants G75A, G77A, and P79A showed a 10- to 20-fold decrease in affinity for AdoMet, whereas D71A exhibited a 10-fold increase, which supports the notion of an important role for these highly conserved residues in cofactor binding. Mutation G73A, also affecting the GxGxG AdoMet-binding signature motif $\left({ }^{73} \mathrm{GxGxG}^{77}\right)$ (Fig. 4A), had a comparatively minor effect, which may explain the nearly wild-type phenotype associated with this mutation (Table 3). Interestingly, protein R139A showed a wild-type affinity, indicating that residue R139 is not involved in AdoMet binding. This result, together with the peculiar orientation of R139 in the AdoMet binding pocket and the finding that protein R139 was unable to modify 16S rRNA, supports our proposal of a catalytic role for this residue. Since mutant R139K was not fully active, it seems that lysine can only partially replace the function of R139. Change to adenine of H53 and D56, two residues structurally close to $\mathrm{R} 139$, had a greater effect on rRNA modification than on the AdoMet binding activity of RsmG. Thus, they may contribute, together with R139, to provide a favorable electrostatic environment for the methyl transfer reaction. Positively charged residues on the protein surface around the active site (K100/R101, $\mathrm{R} 123, \mathrm{~K} 165$, and R197) might play a role in the binding of the incoming 530 loop since their change to alanine impairs the modification function of RsmG. However, we cannot rule out that R197 can participate in G527 coordination since a structural analysis has indicated that its homolog in T. thermophilus (R221) comes close to the R139-D56-H53 cluster (R158-D68-H65 in T. thermophilus), probably as a result of structural rearrangements in the protein upon cofactor binding (Gregory et al. 2009). Overall, our data demonstrate for the first time the critical importance of some residues in or around the active site of E. coli RsmG (H53, D56, D71, G75, G77, P79, D96, $\mathrm{K} 100 / \mathrm{R} 101, \mathrm{R} 139$, and R197) for the $\mathrm{m}^{7} \mathrm{G}$ modification process. Note that mutations affecting the residues corresponding to H53, G77, P79, and R101 in E. coli RsmG give rise to streptomycin resistance in Streptomyces coelicolor (H53), B. subtilis (G77 and R101), and Mycobacterium tuberculosis (P79) (Nishimura et al. 2007a,b; Okamoto et al. 2007).

\section{CONCLUDING REMARKS}

Synthesis of rRNA is linked to several global regulatory networks and thus is carefully balanced in response to changing growth conditions. Modification of rRNA is thought to improve the ribosome performance; however, how expression and activity of rRNA modifying enzymes conform to rRNA amounts is a relevant question that remains poorly explored.

RsmG is an AdoMet-dependent methyltransferase responsible for the synthesis of $\mathrm{m}^{7} \mathrm{G} 527$ in the 530 loop of bacterial $16 \mathrm{~S}$ rRNA. The 530 loop region is universally conserved and appears to play a key role in ribosomal accuracy. 
A
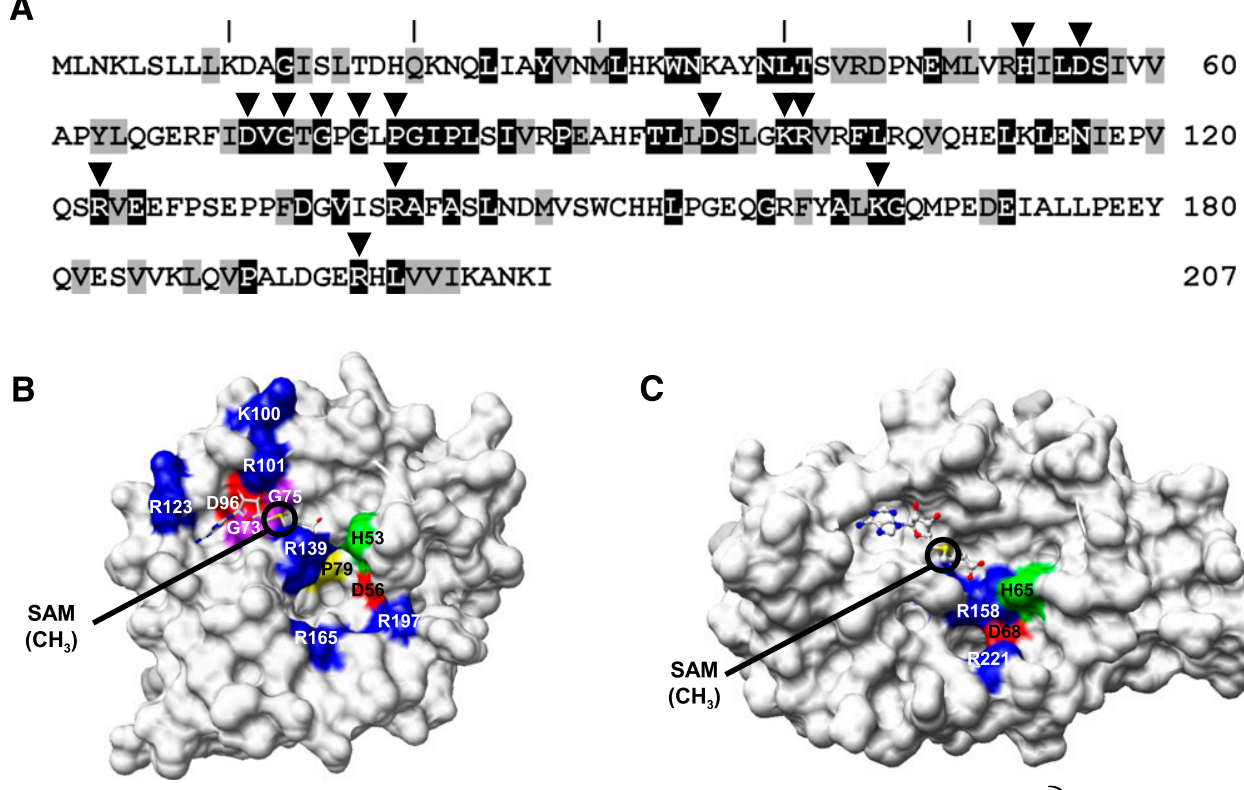

C

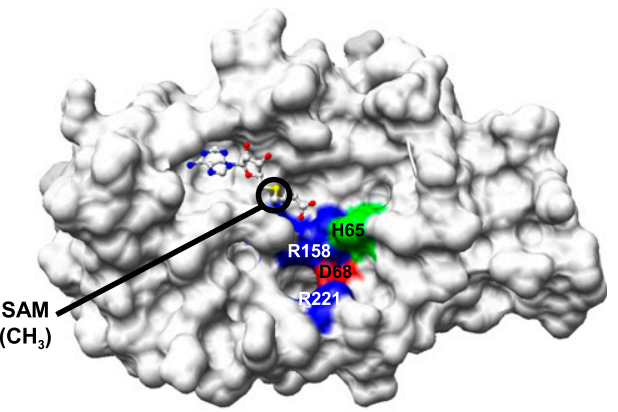

D

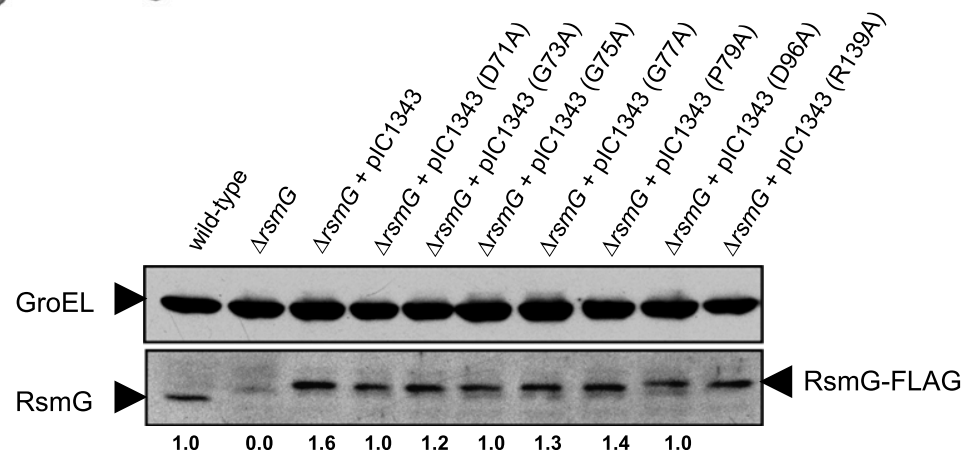

FIGURE 4. Comparative structural analysis of E. coli RsmG. (A) Amino acid sequence of E. coli RsmG. Black and gray boxes highlight conserved and similar residues along the multiple sequence alignment of the RsmG family, respectively. The highly conserved residues selected for experimental characterization because of their proximity to the RsmG active center are marked with black arrowheads. $(B)$ Surface representation of the E. coli RsmG holo model containing the docked AdoMet cofactor and showing the conserved residues selected for alanine scanning. Residues D71 and G77 are not visible in this surface view of the structure. (C) Surface representation of T. thermophilus RsmG complexed with AdoMet (Gregory et al. 2009) (PDB 3G88) displaying the residues homologous to those proposed herein to play a role in the methyl group transfer. $(D)$ Western blot showing the expression level of some RsmG-FLAG mutant proteins used for the in vivo complementation assays $\left(\mathrm{m}^{7} \mathrm{G}\right.$ production and streptomycin resistance). Bands were quantified by densitometry and normalized against GroEL. The intensity ratio is shown below the blot.

Modification $\mathrm{m}^{7} \mathrm{G} 527$ is probably involved in this role since inactivation of RsmG, in certain genetic backgrounds, produces an error-prone phenotype (A Benítez-Páez, M Villarroya, and M-E Armengod, in prep.). Information on the mechanisms regulating RsmG expression and activity has been scarce so far. Here, we confirm that the E. coli rsm $G$ gene is the second member in a bicistronic operon headed by $m n m G$ and demonstrate that $r s m G$ has its own promoter $(r s m G p)$, which allows a noncoordinate regulation of the $m n m G-r s m G$ operon genes. We also show that protein RsmG is almost twofold less stable than MnmG. These results suggest that activity of rsmGp may compensate both the inhibition of the operon promoter $(m n m G p)$ after replication initiation at oriC and the relatively lower stability of RsmG, thus playing an important role during exponential growth. Curiously, activity of $r s m G p$ seems to not be coordinated with transcription initiation of rRNA genes since it increases slightly under conditions that induce ppGpp accumulation and, consequently, repress rRNA synthesis. In contrast, we have found that the levels of the RsmG protein decrease under such conditions. These findings support that mechanisms acting on transcription elongation, translation, or protein stability could be responsible for adjusting RsmG expression to rRNA synthesis. In this respect, we also demonstrate that a putative transcriptional pause signal, which is located between rsmGp and the $r s m G$ ribosome-binding site, works as a weak terminator, a feature that makes it a good candidate to negatively modulate the RsmG expression in response to stress conditions. 


\section{Benítez-Páez et al.}

TABLE 3. Properties of the RsmG mutant proteins

\begin{tabular}{llccc}
\hline Protein $^{\mathrm{a}}$ & $\begin{array}{c}\text { Predicted } \\
\text { function }\end{array}$ & $\begin{array}{c}\text { Low-level Sm } \\
\text { resistance }^{\mathrm{b}}\end{array}$ & $\begin{array}{c}\text { In vivo } \\
\text { activity }^{\mathrm{c}}\end{array}$ & $\begin{array}{c}\text { AdoMet affinity } \\
K_{\mathrm{d}}(\mu \mathrm{M})\end{array}$ \\
\hline Wild type & Catalysis & - & 1.00 & $0.39 \pm 0.02$ \\
H53A & Catalysis & + & $0.28 \pm 0.02$ & $0.77 \pm 0.03$ \\
D56A & SAM binding & ++ & $0.13 \pm 0.03$ & $0.47 \pm 0.17$ \\
D71A & SAM binding & - & 0.00 & $0.04 \pm 0.004$ \\
G73A & SAM binding & ++ & $0.01 \pm 0.09$ & $1.39 \pm 0.20$ \\
G75A & SAM binding & ++ & 0.00 & $9.65 \pm 1.50$ \\
G77A & SAM binding & ++ & $0.05 \pm 0.05$ & $4.09 \pm 0.16 \pm 1.10$ \\
P79A & SAM binding & ++ & 0.00 & $\mathrm{ND}$ \\
D96A & RNA binding & ++ & 0.00 & $\mathrm{ND}$ \\
K100A/R101A & RNA binding & - & $0.84 \pm 0.04$ & $\mathrm{ND}$ \\
R123A & Catalysis & ++ & 0.00 & $0.32 \pm 0.03$ \\
R139A & Catalysis & + & $0.51 \pm 0.06$ & $\mathrm{ND}$ \\
R139K & RNA binding & - & $0.90 \pm 0.01$ & $\mathrm{ND}$ \\
K165A & RNA binding & ++ & $0.15 \pm 0.05$ & $\mathrm{ND}$ \\
R197A & RNA & \\
\hline
\end{tabular}

${ }^{\mathrm{a}}$ RsmG-Flag proteins, expressed in IC5695 ( $\Delta$ rsmG) from pIC1343 (pBAD-TOPO-rsmG-flag) and derivatives, were tested in the in vivo complementation assays (streptomycin resistance and rRNA modification activity), whereas RsmG-His proteins, overexpressed in BL21(DE3) harboring plC1405 (pET15b-rsmG-his) and derivatives, were used for the AdoMet binding assay.

${ }^{b}$ According to growth on LAT plates containing $20 \mu \mathrm{g}$ streptomycin $(\mathrm{Sm})$, resistance was categorized as null $(-)$, intermediate $(+)$, and full $(++)$.

${ }^{\mathrm{C}}$ The modification activity of each RsmG mutant protein in vivo is expressed in relation to the wild-type protein as described in the Materials and Methods section. The data are the mean \pm SEM of two experiments.

${ }^{d}(N D)$ not determined. Introduction of mutation D96A in RsmG-His resulted in a very unstable protein when overexpressed. which is necessary to understand how cells adjust rRNA modification to different physiological conditions.

\section{MATERIALS AND METHODS}

\section{Bacterial strains, plasmids, media, and DNA manipulations}

E. coli strains and plasmids used in this work are listed in Table 4. P1 transduction (Miller 1992) was used to introduce the desired allele into the chromosome of the recipient strain. LBT (Luria Bertani broth containing $40 \mu \mathrm{g} / \mathrm{mL}$ thymine) and LAT (LBT containing $20 \mathrm{~g}$ of Difco agar per liter) were used for routine cultures and plating of E. coli. YM9 buffer contained (per liter) $11 \mathrm{~g}$ of $\mathrm{Na}_{2} \mathrm{HPO}_{4} \cdot 7 \mathrm{H}_{2} \mathrm{O}, 3 \mathrm{~g}$ of $\mathrm{KH}_{2} \mathrm{PO}_{4}, 1 \mathrm{~g}$ of $\mathrm{NH}_{4} \mathrm{Cl}$, and $5 \mathrm{~g}$ of $\mathrm{NaCl}$. M9glu was YM9 supplemented with $1 \%$ glucose, $0.2 \%$ yeast extract, $0.2 \%$ casamino acids, $1 \mu \mathrm{g} / \mathrm{mL}$ vitamin B1, and $20 \mu \mathrm{g} / \mathrm{mL}$ thymine. M9gly was YM9 supplemented with $0.5 \%$ glycerol, 50 $\mu \mathrm{g} / \mathrm{mL}$ leucine, $1 \mu \mathrm{g} / \mathrm{mL}$ vitamin $\mathrm{B} 1$, and 20 $\mu \mathrm{g} / \mathrm{mL}$ thymine. Antibiotics were added as recommended (Miller 1992), unless otherwise stated. Standard procedures were followed for DNA manipulations. All constructs were verified by DNA sequencing. Transcriptional lac $Z$ fusions were constructed by amplifying

The efficiency of the rRNA modification process mediated by RsmG may depend not only on the RsmG concentration within the cell but also on the biochemical properties and enzymatic mechanism of the protein. Information about the mechanism of $\mathrm{m}^{7} \mathrm{G}$ formation in RNA is limited. This nucleoside is also found at position 1405 in $16 \mathrm{~S}$ rRNA, at position 46 of tRNA from Bacteria and Eukaryota, and in the cap structure of eukaryotic mRNA. Comparisons of available structures of enzymes catalyzing $\mathrm{m}^{7} \mathrm{G}$ synthesis have revealed a surprising diversity of active sites and binding modes for the same basic modification reaction (Husain et al. 2010), suggesting that these methyltransferases may use different molecular mechanisms. To gain insights into the mechanism of $\mathrm{m}^{7} \mathrm{G}$ formation by RsmG, we built a sequence profile of the RsmG family from 27 sequences representative of most bacterial groups. Based on both this profile and structural comparisons, a series of conserved residues was mutated, and the abilities of the resulting proteins to confer streptomycin resistance, to modify rRNA, and to bind AdoMet were explored. Our data demonstrate for the first time the critical importance of some residues located at the active site of E. coli RsmG for the $\mathrm{m}^{7} \mathrm{G}$ modification process and suggest that they play a role in rRNA binding and catalysis.

In conclusion, our work provides valuable information on the molecular bases controlling RsmG levels and activity, specific DNA fragments with primers incorporating $X$ hoI restriction sites. PCR products were purified, XhoI digested, and inserted into XhoI-linearized pIC552 plasmid (Macian et al. 1994).

\section{Enzyme assays}

$\beta$-galactosidase and $\beta$-lactamase activities were determined as described previously (Andrup et al. 1988; Miller 1992) with slight modifications. Cultures were maintained for at least 5-10 generations in exponential growth by repeated dilution with the same medium before being assayed. $\beta$-galactosidase assays were performed on SDSchloroform-permeabilized cells. Special precautions for very low $\beta$-galactosidase activities were taken (Elseviers et al. 1984; Bregeon et al. 2001). $\beta$-galactosidase activity of each transcriptional fusion carried on pIC552 was normalized to the $\beta$-lactamase activity encoded by the same plasmid. Data were compared for statistical significance using a $t$-test with Welch's correction.

\section{Prediction of DNA regulatory elements} rsmG operon (positions 3,921,080 to 3,924,034 of the NC_000913 GenBank entry) was submitted to the Neural Network Promoter Prediction (Reese 2001) and BPROM (http:// www.softberry.com/berry.phtml) web servers. Predictions from both servers are plotted in Figure 2A (at top). Prediction of RNA secondary structure in the E. coli mnmG-rsmG intergenic region (positions 1891-1953) was performed using the mfold server (Zuker 2003).
A sequence between positions -378 and 2577 of the $m n m G$ - 
TABLE 4. List of strains and plasmids used in this study

\begin{tabular}{|c|c|c|c|}
\hline Designation & Description & Construction & Origin \\
\hline & Strains & & \\
\hline BW25113 & $\begin{array}{c}\mathrm{F}^{-} \Delta(\operatorname{araD}-\mathrm{araB}) 567, \Delta / a c Z 4787(:: \mathrm{rrnB}-3) \\
\lambda^{-}, \mathrm{rph}-1, \Delta(\text { rhaD-rhaB)568, hsdR514 }\end{array}$ & & Baba et al. 2006 \\
\hline DEV16 & $\mathrm{F}^{-}$thi-1 rel-1 spoT1 lacZ105 UAG val ${ }^{R}$ mnmEQ192X & & Elseviers et al. 1984 \\
\hline MC1000 & $\begin{array}{l}\mathrm{F}^{-} \text {araD139 } \Delta(\text { ara-leu }) 7679 \text { galUK } \Delta(\text { lac }) X 74 \\
\text { thi rpsL150 relA1 spoT1 }\end{array}$ & & \\
\hline MC4100 & $\begin{array}{l}\mathrm{F}^{-} \Delta(\text { arg-lac) U169 araD139 rpsL150 ptsF25 } \\
\text { fibB5301 rbsR deoC relA1 }\end{array}$ & & \\
\hline RH90 & MC4100 rрoS359::Tn10 & & Muffler et al. 1996 \\
\hline TOP10 & & & Invitrogen \\
\hline BL21(DE3) & & & Novagen \\
\hline IC4639 & DEV16 mnmE $E^{+} b g l$ & & Yim et al. 2006 \\
\hline IC5678 & BW25113 rsmG::kan a & & Baba et al. 2006 \\
\hline IC5695 & IC4639 rsmG::kan ${ }^{a}$ & P1 $(\mathrm{IC} 5678) \times \mathrm{IC} 4639$ & This study \\
\hline IC5831 & BW25113 mnmG::kan & & Baba et al. 2006 \\
\hline IC5930 & NECB1 mnmG::Tn 10 (clone 4) & & Bregeon et al. 2001 \\
\hline IC5931 & NECB1 mnmG::Tn 10 (clone 8) & & Bregeon et al. 2001 \\
\hline IC5932 & NECB1 mnmG::Tn 10 (clone 10) & & Bregeon et al. 2001 \\
\hline IC5933 & IC4639 mnmG::Tn10 & P1 (IC5930) $\times$ IC4639 & This study \\
\hline IC5934 & IC4639 mnmG::Tn10 & P1 $($ IC5931) $\times$ IC4639 & This study \\
\hline IC5935 & IC4639 mnmG::Tn10 & P1 $($ IC5932) $\times$ IC4639 & This study \\
\hline IC5936 & $\begin{array}{l}\text { IC4639 mnmG::kan } \\
\text { Plasmids }\end{array}$ & P1 $($ IC5831) $\times$ IC4639 & This study \\
\hline pBAD-TOPO & $\begin{array}{l}\text { Vector used for the production of C-terminally } \\
\text { FLAG-tagged proteins under } \mathrm{P}_{\mathrm{BAD}} \text { and AraC control }\end{array}$ & & Invitrogen \\
\hline plC552 & Vector for lacZ transcriptional fusions & & Macian et al. 1994 \\
\hline pET15b & $\begin{array}{l}\text { Vector used for the production of C-terminally } \\
\text { His-tagged proteins under T7 promoter control }\end{array}$ & & Novagen \\
\hline plC1343 & pBAD-TOPO-rsmG-flag & & This study \\
\hline plC1344 & plC552 + fragment $1544-1953^{b}$ & & This study \\
\hline plC1345 & pBAD-TOPO-mnmG & & This study \\
\hline plC1371 & plC552 + fragment $1544-1890^{\mathrm{b}}$ & & This study \\
\hline plC1372 & plC552 + fragment $1739-1953^{b}$ & & This study \\
\hline plC1373 & plC552 + fragment $1739-1890^{\mathrm{b}}$ & & This study \\
\hline $\mathrm{plC} 1374$ & plC552 + fragment $(-162)$ to $(-1)^{\mathrm{b}}$ & & This study \\
\hline plC1405 & pET15b-rsmG-his & & This study \\
\hline plC1460 & plC552 + fragment $1838-1890^{b}$ & & This study \\
\hline plC1461 & plC552 + fragment $1739-1842^{b}$ & & This study \\
\hline plC1664 & plC552 + fragment $1838-2653^{b}$ & & This study \\
\hline plC1665 & plC552 + fragment $1891-2653^{b}$ & & This study \\
\hline plC1686 & pBAD-TOPO-rsmG & & This study \\
\hline
\end{tabular}

\section{Sequence and comparative structural analysis}

Phylogenetic distribution of RsmG was studied with the STRING server (von Mering et al. 2007). A general procedure for detecting functional residues in protein families based on sequence analysis was used (Benítez-Páez et al. 2011). Briefly, 27 protein sequences belonging to organisms of the most representative eubacterial groups were retrieved from Uniprot database (Uniprot Consortium 2010) and aligned using the MUSCLE program and default parameters (Edgar 2004). Then, a Hidden Markov Model (HMM)-based profile was built using HMMER v. 2.3.2 application and default parameters (Eddy 1998). Highly conserved residues of RsmG proteins revealed by the HMM-profile were visualized with the Logomat-P software (Schuster-Bockler and Bateman 2005). To build a model of the E. coli RsmG holoprotein, the apo structure of E. coli RsmG (Uniprot id P0A6U5) was used as bait in a Blastp search for finding PDB homologues in complex with AdoMet. The RsmG protein from T. thermophilus (Gregory et al. 2009) (PDB id 3G88) was the best match obtained containing the cofactor $(\sim 31 \%$ identity). Alpha carbons of the glycines composing the canonical AdoMet-binding motif GxGxG were used to superimpose both structures with DeepView (Guex and Peitsch 1997). RSM deviation between the AdoMet motifs was calculated to be $0.16 \AA$. Molecular graphics images were produced using the UCSF Chimera package from the Resource for Biocomputing, Visualization, and Informatics at the University of California, San Francisco, California (Pettersen et al. 2004). 


\section{Protein techniques and production of antisera}

The E. coli rsmG gene was cloned into the pBAD-TOPO vector, generating plasmid pIC1343. The RsmG-Flag recombinant protein expressed from this plasmid in TOP10 cells was purified by affinity chromatography (Anti-Flag M2 Affinity Gel, Sigma) and used to inoculate New Zealand rabbits. Before use, the antiserum was purified on nitrocellulose-bound RsmG-Flag. Production of the anti-MnmG antibody has been described previously (Yim et al. 2006). Anti-GroEL monoclonal mouse antibody was purchased from Calbiochem (Merck KGaA).

To determine the RsmG levels in strains carrying different chromosomal rsmG alleles (IC4639 and its derivatives IC5695, IC5933, IC5934, IC5935, and IC5936), cells were maintained under steady-state exponential growth for several generations, allowed to grow to the late exponential phase $\left(\mathrm{OD}_{600} \sim 0.7-0.8\right)$, collected, and disrupted by sonication. Soluble fractions were recovered by centrifugation at $16000 \mathrm{~g}$ for $20 \mathrm{~min}$ at $4^{\circ} \mathrm{C}$. Protein concentration was measured by the Bradford assay (Bio-Rad Protein Assay) using bovine serum albumin (BSA) as a standard. Then, $200 \mu \mathrm{g}$ of each soluble protein extract were analyzed by SDS-PAGE. Proteins were transferred to a nitrocellulose membrane and incubated overnight with anti-MnmG (1/5000) and anti-RsmG (1/5000) or for 30 min with anti-GroEL $(1 / 20,000)$. Membranes were washed twice with TBS $+0.1 \%$ Igepal and subsequently incubated with an anti-IgG peroxidase-conjugated secondary antibody (1/5000) for $1 \mathrm{~h}$. Finally, detection of the native MnmG, RsmG, and GroEL proteins was performed with ECL Western Blotting Detection Reagents (GE Healthcare) following the manufacturer's instructions.

\section{Protein half-life determination}

The IC5936 and IC5695 strains carrying pIC1345 and pIC1686, respectively, were cultured in LBT media with ampicillin $(100 \mu \mathrm{g} /$ $\mathrm{mL})$ at $37^{\circ} \mathrm{C}$. Overnight cultures were diluted $1 / 100$ in the same media supplemented with $0.02 \%$ L-Arabinose (Sigma). Cultures were incubated with shaking at $37^{\circ} \mathrm{C}$ for $2 \mathrm{~h}$. The AraC- $\mathrm{P}_{\mathrm{BAD}}$ controlled expression of the $m n m G$ or $r s m G$ genes was stopped by a brief centrifugation at $3000 \mathrm{~g}$ for $10 \mathrm{~min}$ followed by resuspension of the cells in the same volume of prewarmed LBT supplemented with ampicillin and $1 \%$ glucose. Cultures continued to grow at $37^{\circ} \mathrm{C}$, and samples were taken at $0,15,30,60,90,120,150$, and $180 \mathrm{~min}$ after addition of glucose. Samples were stored on ice until processed for Western blot analysis. Band intensities were measured by using the ImageQuant TL v2005 software (Amersham). The signal was normalized by using GroEL as a loading control. Fitting of the exponential decay and half-life determination were performed with GraphPad Prism v4.0 (GraphPad Software Inc.).

\section{In vivo complementation assays}

Strain IC5695 $(\Delta r s m G)$ carrying pBAD-TOPO or pBAD-TOPO derivatives encoding C-terminal FLAG-tagged RsmG proteins was grown in the absence of the arabinose inducer to achieve recombinant protein expression levels close to the native protein level. For the streptomycin-resistance assay, an aliquot of each culture at an $\mathrm{OD}_{600}$ of 0.4 was diluted $1 / 10$ and spotted in triplicate on LAT plates supplemented with $20 \mu \mathrm{g} / \mathrm{mL}$ of streptomycin. Cell growth was evaluated after incubation of the plates for $12-16 \mathrm{~h}$ at $37^{\circ} \mathrm{C}$.
To test the ability of RsmG proteins expressed from pBADTOPO and pIC552 derivatives to modify $16 \mathrm{~S}$ rRNA, $100 \mathrm{~mL}$ cultures grown as above, until an $\mathrm{OD}_{600}$ value of $\sim 0.8$, were processed for extraction of $16 \mathrm{~S}$ rRNA. Presence of $\mathrm{m}^{7} \mathrm{G}$ in this rRNA was analyzed by HPLC.

\section{Analysis of the methylation status of 165 rRNA}

Total RNA was obtained as described previously (Emilsson and Kurland 1990; Benítez-Páez et al. 2010). The specific 16S rRNA fraction was purified by the Chaplet Column Chromatography method (Suzuki and Suzuki 2007) using the DNA probe biotin $_{5}{ }^{\prime}$ CAGTCTTCGTCCAGGGGGCCGCCTTCGCCACC, complementary to the 721-752 region of the E. coli $16 \mathrm{~S}$ rRNA and immobilized in a HiTrap Streptavidin HP column (GE Healthcare). Afterward, $25 \mu \mathrm{g}$ of the purified rRNA were digested overnight with nuclease P1 (Sigma) and subsequently treated with E. coli alkaline phosphatase (Sigma) for $2 \mathrm{~h}$. The resulting nucleosides were analyzed by HPLC using a Develosil $5 \mu$ RP-AQUEOUS C-30 column (Phenomenex) with a gradient elution program in which buffer A had 2.5\% methanol, $10 \mathrm{mM} \mathrm{NH}_{4} \mathrm{H}_{2} \mathrm{PO}_{4}, \mathrm{pH}$ 5.3, while buffer $\mathrm{B}$ had $25 \%$ methanol, $10 \mathrm{mM} \mathrm{NH}_{4} \mathrm{H}_{2} \mathrm{PO}_{4}, \mathrm{pH}$ 5.1. The $\mathrm{m}^{7} \mathrm{G}, \mathrm{m}^{5} \mathrm{C}$, and $\mathrm{m}^{2} \mathrm{G}$ nucleosides were monitored by using commercial standards (Sigma). In the assays shown in Table 1, the relative modification activity of each RsmG mutant protein was quantified in terms of the peak area ratio as follows: $\left[\left(\mathrm{m}^{7} \mathrm{G} /\right.\right.$ $\left.\left.\left.\mathrm{m}^{2} \mathrm{G}\right)_{\mathrm{RsmG}}-\left(\mathrm{m}^{7} \mathrm{G} / \mathrm{m}^{2} \mathrm{G}\right)_{\text {null }}\right)\right] /\left[\left(\mathrm{m}^{7} \mathrm{G} / \mathrm{m}^{2} \mathrm{G}\right)_{\text {wild-type }}-\left(\mathrm{m}^{7} \mathrm{G} / \mathrm{m}^{2}-\right.\right.$ $\left.\left.\mathrm{G})_{\text {null }}\right)\right]$, where the subscript indicates the peak areas observed in the null mutant strain harboring the empty vector ("null") or the recombinant plasmid expressing the tested RsmG ("RsmG") or wild-type ("wild-type") protein. In the assays shown in Figure 3, the $\mathrm{m}^{7} \mathrm{G}$ levels were normalized to those of $\mathrm{m}^{2} \mathrm{G}$ or $\mathrm{m}^{5} \mathrm{C}$, obtaining similar final values in both cases.

\section{S-adenosyl-L-methionine (AdoMet) binding assay}

C-terminal 6His-tagged RsmG proteins, overexpressed from pET15b derivatives, were tested for AdoMet binding by Surface Plasmon Resonance (SPR) on a Biacore T100 (GE Healthcare). A monoclonal anti-His-tag antibody was immobilized on a CM5 sensor chip using the Amine Coupling Kit (Biacore, GE Healthcare). The best protein immobilization conditions were achieved using a low-salt PBS buffer solution $(0.25 \times)$ at $\mathrm{pH}$ 6.0. Then, $7 \mu \mathrm{g}$ of protein were immobilized for $150 \mathrm{sec}$ with flux at $10 \mu \mathrm{L} / \mathrm{min}$. Different concentrations of AdoMet (New England Biolabs) were tested (ranging from $100 \mathrm{nM}$ to $10 \mu \mathrm{M}$ ). The contact time was set at $40 \mathrm{sec}$ at the same flux as before. AdoMet affinities were calculated using the Biacore T100 Evaluation Software, V2.0 (Biacore, GE Healthcare).

\section{SUPPLEMENTAL MATERIAL}

Supplemental material is available for this article.

\section{ACKNOWLEDGMENTS}

We thank Dr. D. Brégeon, and the National BioResource Project (NIG, Japan) for providing strains used in this study. This work has been supported by the Spanish Ministry of Science and Innovation (BFU2007-66509; BFU2010-19737) and the Generalitat 
Valenciana (ACOMP/2010/236) to M.-E.A. and a PhD fellowship from the Centro de Investigación Príncipe Felipe to A.B.-P.

Received August 11, 2011; accepted January 5, 2012.

\section{REFERENCES}

Andrup L, Atlung T, Ogasawara N, Yoshikawa H, Hansen FG. 1988. Interaction of the Bacillus subtilis DnaA-like protein with the Escherichia coli DnaA protein. J Bacteriol 170: 1333-1338.

Baba T, Ara T, Hasegawa M, Takai Y, Okumura Y, Baba M, Datsenko KA, Tomita M, Wanner BL, Mori H. 2006. Construction of Escherichia coli K-12 in-frame, single-gene knockout mutants: The Keio collection. Mol Syst Biol 2: 2006.0008. doi: 10.1038/ msb4100050.

Benítez-Páez A, Villarroya M, Douthwaite S, Gabaldon T, Armengod ME. 2010. YibK is the $2^{\prime}$-O-methyltransferase TrmL that modifies the wobble nucleotide in Escherichia coli tRNA ${ }^{\text {Leu }}$ isoacceptors. RNA 16: 2131-2143.

Benítez-Páez A, Cardenas-Brito S, Gutierrez AJ. 2011. A practical guide for the computational selection of residues to be experimentally characterized in protein families. Brief Bioinform doi: 10.1093/bib/bbr052.

Bernstein JA, Khodursky AB, Lin PH, Lin-Chao S, Cohen SN. 2002. Global analysis of mRNA decay and abundance in Escherichia coli at single-gene resolution using two-color fluorescent DNA microarrays. Proc Natl Acad Sci 99: 9697-9702.

Bregeon D, Colot V, Radman M, Taddei F. 2001. Translational misreading: A tRNA modification counteracts a +2 ribosomal frameshift. Genes Dev 15: 2295-2306.

Carter AP, Clemons WM, Brodersen DE, Morgan-Warren RJ, Wimberly BT, Ramakrishnan V. 2000. Functional insights from the structure of the $30 \mathrm{~S}$ ribosomal subunit and its interactions with antibiotics. Nature 407: 340-348.

Eddy SR. 1998. Profile hidden Markov models. Bioinformatics 14: 755-763.

Edgar RC. 2004. MUSCLE: A multiple sequence alignment method with reduced time and space complexity. BMC Bioinformatics 5: 113. doi: 10.1186/1471-2105-5-113.

Elseviers D, Petrullo LA, Gallagher PJ. 1984. Novel E. coli mutants deficient in biosynthesis of 5-methylaminomethyl-2-thiouridine. Nucleic Acids Res 12: 3521-3534.

Emilsson V, Kurland CG. 1990. Growth rate dependence of transfer RNA abundance in Escherichia coli. EMBO J 9: 4359-4366.

Gregory ST, Demirci H, Belardinelli R, Monshupanee T, Gualerzi C, Dahlberg AE, Jogl G. 2009. Structural and functional studies of the Thermus thermophilus $16 \mathrm{~S}$ rRNA methyltransferase RsmG. RNA 15: $1693-1704$.

Guex N, Peitsch MC. 1997. SWISS-MODEL and the SwissPdbViewer: An environment for comparative protein modeling. Electrophoresis 18: 2714-2723.

Gummesson B, Magnusson LU, Lovmar M, Kvint K, Persson O, Ballesteros M, Farewell A, Nystrom T. 2009. Increased RNA polymerase availability directs resources towards growth at the expense of maintenance. EMBO J 28: 2209-2219.

Hansen F, Koefoed S, von Meyenburg K, Atlung T. 1981. Transcription and translation events in the oriC region of the $\mathrm{E}$. coli chromosome. ICN-UCLA Symp Mol Cell Biol 22: 37-55.

Husain N, Tkaczuk KL, Tulsidas SR, Kaminska KH, Cubrilo S, Maravic-Vlahovicek G, Bujnicki JM, Sivaraman J. 2010. Structural basis for the methylation of G1405 in 16S rRNA by aminoglycoside resistance methyltransferase Sgm from an antibiotic producer: A diversity of active sites in $77 \mathrm{G}$ methyltransferases. Nucleic Acids Res 38: 4120-4132.

Ishihama Y, Schmidt T, Rappsilber J, Mann M, Hartl FU, Kerner MJ, Frishman D. 2008. Protein abundance profiling of the Escherichia coli cytosol. BMC Genomics 9: 102. doi: 10.1186/1471-2164-9-102.
Jin DJ, Cagliero C, Zhou YN. 2011. Growth rate regulation in Escherichia coli. FEMS Microbiol Rev doi: 10.1111/j.1574-6976.2011.00279.x.

Keener J, Nomura M. 1996. Regulation of ribosome synthesis. In Escherichia coli and Salmonella: Cellular and molecular biology (ed. F Neidhardt et al.), pp. 1417-1431. ASM Press, Washington DC.

Kolling R, Gielow A, Seufert W, Kucherer C, Messer W. 1988. AsnC, a multifunctional regulator of genes located around the replication origin of Escherichia coli, oriC. Mol Gen Genet 212: 99-104.

Macian F, Perez-Roger I, Armengod ME. 1994. An improved vector system for constructing transcriptional lacZ fusions: Analysis of regulation of the dnaA, dnaN, recF, and gyrB genes of Escherichia coli. Gene 145: 17-24.

Magnusson LU, Farewell A, Nystrom T. 2005. ppGpp: A global regulator in Escherichia coli. Trends Microbiol 13: 236-242.

Miller JH. 1992. A short course in bacterial genetics: A laboratory manual and handbook for Escherichia coli and related bacteria. Cold Spring Harbor Laboratory Press, Cold Spring Harbor, NY.

Moukadiri I, Prado S, Piera J, Velazquez-Campoy A, Bjork GR, Armengod ME. 2009. Evolutionarily conserved proteins MnmE and GidA catalyze the formation of two methyluridine derivatives at tRNA wobble positions. Nucleic Acids Res 37: 7177-7193.

Muffler A, Fischer D, Altuvia S, Storz G, Hengge-Aronis R. 1996. The response regulator RssB controls stability of the $\sigma^{\mathrm{S}}$ subunit of RNA polymerase in E. coli. EMBO J 15: 1333-1339.

Nishimura K, Hosaka T, Tokuyama S, Okamoto S, Ochi K. 2007a. Mutations in rsmG, encoding a $16 \mathrm{~S}$ rRNA methyltransferase, result in low-level streptomycin resistance and antibiotic overproduction in Streptomyces coelicolor A3(2). J Bacteriol 189: 38763883.

Nishimura K, Johansen SK, Inaoka T, Hosaka T, Tokuyama S, Tahara Y, Okamoto S, Kawamura F, Douthwaite S, Ochi K. 2007b. Identification of the RsmG methyltransferase target as 16S rRNA nucleotide G527 and characterization of Bacillus subtilis rsmG mutants. J Bacteriol 189: 6068-6073.

Ochi K, Kim JY, Tanaka Y, Wang G, Masuda K, Nanamiya H, Okamoto S, Tokuyama S, Adachi Y, Kawamura F. 2009. Inactivation of KsgA, a 16S rRNA methyltransferase, causes vigorous emergence of mutants with high-level kasugamycin resistance. Antimicrob Agents Chemother 53: 193-201.

Ogawa T, Okazaki T. 1991. Concurrent transcription from the gid and mioC promoters activates replication of an Escherichia coli minichromosome. Mol Gen Genet 230: 193-200.

Ogawa T, Okazaki T. 1994. Cell cycle-dependent transcription from the gid and mioC promoters of Escherichia coli. J Bacteriol 176: 1609-1615.

Ogle JM, Ramakrishnan V. 2005. Structural insights into translational fidelity. Annu Rev Biochem 74: 129-177.

Ogle JM, Carter AP, Ramakrishnan V. 2003. Insights into the decoding mechanism from recent ribosome structures. Trends Biochem Sci 28: 259-266.

Okamoto S, Tamaru A, Nakajima C, Nishimura K, Tanaka Y, Tokuyama S, Suzuki Y, Ochi K. 2007. Loss of a conserved 7-methylguanosine modification in 16S rRNA confers low-level streptomycin resistance in bacteria. Mol Microbiol 63: 10961106.

Pettersen EF, Goddard TD, Huang CC, Couch GS, Greenblatt DM, Meng EC, Ferrin TE. 2004. UCSF Chimera-a visualization system for exploratory research and analysis. J Comput Chem 25: 16051612.

Potrykus K, Cashel M. 2008. (p)ppGpp: Still magical? Annu Rev Microbiol 62: 35-51.

Purta E, van Vliet F, Tricot C, De Bie LG, Feder M, Skowronek K, Droogmans L, Bujnicki JM. 2005. Sequence-structure-function relationships of a tRNA (m7G46) methyltransferase studied by homology modeling and site-directed mutagenesis. Proteins 59: $482-488$.

Reese MG. 2001. Application of a time-delay neural network to promoter annotation in the Drosophila melanogaster genome. Comput Chem 26: 51-56. 


\section{Benítez-Páez et al.}

Romanowski MJ, Bonanno JB, Burley SK. 2002. Crystal structure of the Escherichia coli glucose-inhibited division protein B (GidB) reveals a methyltransferase fold. Proteins 47: 563-567.

Schubert HL, Blumenthal RM, Cheng X. 2003. Many paths to methyltransfer: A chronicle of convergence. Trends Biochem Sci 28: 329-335.

Schuster-Bockler B, Bateman A. 2005. Visualizing profile-profile alignment: Pairwise HMM logos. Bioinformatics 21: 2912-2913.

Srivatsan A, Wang JD. 2008. Control of bacterial transcription, translation, and replication by (p)ppGpp. Curr Opin Microbiol 11: $100-105$.

Suzuki T, Suzuki T. 2007. Chaplet column chromatography: Isolation of a large set of individual RNAs in a single step. Methods Enzymol 425: 231-239.

Theisen PW, Grimwade JE, Leonard AC, Bogan JA, Helmstetter CE. 1993. Correlation of gene transcription with the time of initiation of chromosome replication in Escherichia coli. Mol Microbiol 10: $575-584$.

Uniprot Consortium. 2010. The Universal Protein Resource (UniProt) in 2010. Nucleic Acids Res 38: D142-D148. von Mering C, Jensen LJ, Kuhn M, Chaffron S, Doerks T, Kruger B, Snel B, Bork P. 2007. STRING 7-recent developments in the integration and prediction of protein interactions. Nucleic Acids Res 35: D358-D362.

von Meyenburg K, Jorgensen BB, Nielsen J, Hansen FG. 1982. Promoters of the atp operon coding for the membrane-bound ATP synthase of Escherichia coli mapped by Tn10 insertion mutations. Mol Gen Genet 188: 240-248.

Walker JE, Saraste M, Gay NJ. 1984. The unc operon. Nucleotide sequence, regulation, and structure of ATP-synthase. Biochim Biophys Acta 768: 164-200.

Wong SY, Lee JS, Kwak HK, Via LE, Boshoff HI, Barry CE 3rd. 2011. Mutations in gidB confer low-level streptomycin resistance in Mycobacterium tuberculosis. Antimicrob Agents Chemother 55: 2515-2522.

Yim L, Moukadiri I, Bjork GR, Armengod ME. 2006. Further insights into the tRNA modification process controlled by proteins $\mathrm{MnmE}$ and GidA of Escherichia coli. Nucleic Acids Res 34: 5892-5905.

Zuker M. 2003. Mfold web server for nucleic acid folding and hybridization prediction. Nucleic Acids Res 31: 3406-3415. 

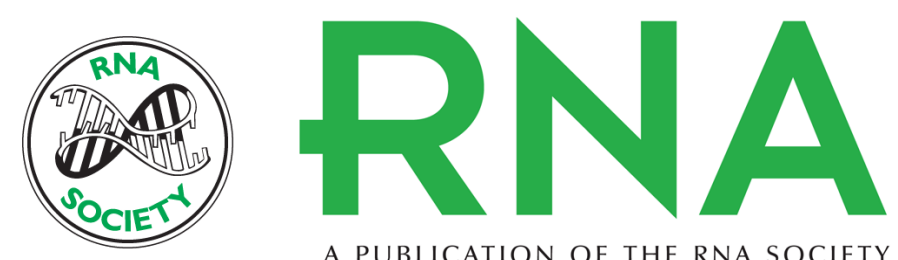

A PUBLICATION OF THE RNA SOCIETY

\section{Regulation of expression and catalytic activity of Escherichia coli RsmG methyltransferase}

Alfonso Benítez-Páez, Magda Villarroya and M.-Eugenia Armengod

RNA 2012 18: 795-806 originally published online February 15, 2012

Access the most recent version at doi:10.1261/rna.029868.111

Supplemental
Material http://rnajournal.cshlp.org/content/suppl/2012/01/31/rna.029868.111.DC1

References This article cites 46 articles, 11 of which can be accessed free at:

http://rnajournal.cshlp.org/content/18/4/795.full.html\#ref-list-1

License

Email Alerting Receive free email alerts when new articles cite this article - sign up in the box at the Service top right corner of the article or click here. 